\title{
Limits on CP Violation from Electric Dipole Moments
}

\author{
Michael V. Romalis \\ Department of Physics, Princeton University, Princeton, NJ 08544
}

\begin{abstract}
Searches for a permanent electric dipole moment (EDM) provide a unique constraint on violation of CP symmetry beyond the Standard Model. Recent experiments improved the limits on EDMs of ${ }^{199} \mathrm{Hg}$ and the electron. Combined with the limit on the neutron EDM and recent advances in theoretical interpretation, they set very tight constraints on CP violation in Supersymmetry. Many new experimental ideas are currently being explored, promising to improve the EDM limits by several orders of magnitude.
\end{abstract}

\section{Introduction}

The search for a permanent electric dipole moment (EDM) started about 50 years ago when Purcell and Ramsey pointed out that while a permanent EDM could be excluded based on symmetry arguments, its existence was ultimately a purely experimental question [1]. Since that time the symmetry arguments against an EDM, namely that it violates parity and time reversal symmetries, have been eliminated, but no EDMs have been found. EDM searches continue today and they play an important role in shaping our understanding of symmetries of physics laws.

The electric dipole moment $d$ conceptually is a very simple quantity that can be introduced in complete analogy to the familiar magnetic dipole moment $\mu$

$$
H=-(d \mathbf{E}+\mu \mathbf{B}) \cdot \frac{\mathbf{S}}{S}
$$

where $\mathbf{S}$ is the spin of the particle. However, because the electric field $\mathbf{E}$, unlike $\mathbf{B}$, is a polar vector, a non-zero EDM violates both parity and time reversal symmetries. In high energy physics one usually talks about $\mathrm{CP}$ violation instead of $\mathrm{T}$ violation, but since nearly all theories considered today are invariant under CPT these two symmetries are virtually equivalent. Since the discovery of $\mathrm{CP}$ violation in $\mathrm{K}^{0}$ mesons many proposed theories of $\mathrm{CP}$ violation have been eliminated because they predicted EDMs larger than existing limits [2]. The Standard Model is fairly unique in explaining $\mathrm{CP}$ violation in $\mathrm{K}$ and $\mathrm{B}$ mesons while predicting only tiny EDMs, about 5 or more orders of magnitude smaller than the existing limits. Thus, EDM searches provide a unique window on new physics and are not affected by theoretical uncertainties in the size of the Standard Model effects. Present efforts to incorporate the Standard Model into a more general high energy theory often again introduce large EDMs incompatible with existing experimental limits [3,4].

It is instructive to compare the electron EDM limit with the uncertainty in its anomalous magnetic moment, which is one of the most accurately measured physical quantities. The limit on the electron EDM $\left|d_{e}\right|<1.6 \times 10^{-27} e \mathrm{~cm}[5]$ is 13 orders of magnitude smaller than the value of its anomalous magnetic moment $\mu_{a}=\mu_{B}(g-$ $2) / 2=2 \times 10^{-14} e \mathrm{~cm}$ and 5 orders of magnitude smaller than the uncertainty in 


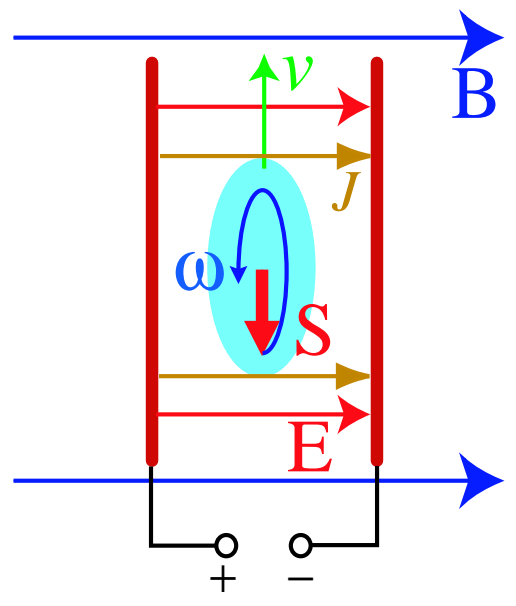

Figure 1: A general setup for an EDM experiment. Spin-polarized atoms are placed in parallel electric and magnetic fields and their spin precession frequency $\omega$ is accurately measured. Also shown are possible leakage currents $J$ and, for beam experiments, velocity $v$ of the atomic beam.

$\mu_{a}, \delta \mu_{a}=1 \times 10^{-22} e \mathrm{~cm}[6]$. This clearly illustrates the experimental and theoretical advantages of measuring and interpreting a quantity that is close to zero.

The basic experimental idea of most EDM searches is to measure the Larmor precession frequency of spin-polarized atoms in parallel electric and magnetic fields

$$
\omega=\frac{2 \mu B+2 d E}{\hbar},
$$

as shown in Fig. 1. The direction of the electric field is frequently reversed and any correlation of the precession frequency with the direction of the electric field serves as a signature of a permanent EDM. The sensitivity of such measurement is fundamentally limited by the spin coherence time $\tau$ and the number $N$ of available atoms as well as the maximum electric field that can be applied without electrical breakdown,

$$
\delta d=\frac{\hbar}{2 E \sqrt{N \tau t}}
$$

where $t$ is the measurement time $(t \gg \tau)$. Many EDM experiments approach this fundamental sensitivity limit and require months, sometimes years, of data accumulation to achieve their statistical sensitivity.

In the same time, as any precision experiment, EDM searches can be quite susceptible to systematic effects. In particular, they depend crucially on the ability to reverse a very strong electric field, often close to the electrical breakdown, without changing the magnetic field by even a slightest, often barely measurable, amount. Two types of systematic effects are of particular concern to most EDM experiments. If the atoms are moving in an electric field they experience a magnetic field in their rest frame $\mathbf{B}_{m}=\mathbf{v} \times \mathbf{E} / c$. This field is odd in $E$ and can mimic an EDM signal. Fortunately the first order frequency shift of this type is significantly suppressed because the electric 
and magnetic fields are nominally parallel and the resulting motional field is orthogonal to the main magnetic field. However higher order effects and misalignments can still lead to substantial systematic effects. The second type of systematic effects comes from leakage currents induced by the electric field. To the extend that the leakage currents follow the lines of the $\mathbf{E}$ field and are sufficiently symmetric, they also do not produce a first order frequency shift. However, leakage currents caused by high voltage are often difficult to measure and control. One of the general methods to combat these types of systematic effects is to introduce a so-called co-magnetometer, for example, a second type of atoms which can measure the magnetic field in the same volume but are not sensitive to the electric dipole moment. The feasibility and effectiveness of the co-magnetometer depends on the details of the experimental apparatus.

\section{Current EDM Experiments}

Currently there are 3 main types of EDM experiments with comparable sensitivity that constrain different combinations of CP-violating interactions. They involve searches for the EDM of the neutron, heavy paramagnetic atoms, and heavy diamagnetic atoms.

The search for a neutron EDM got under way soon after the initial proposal by Ramsey and Purcell and experiments of this type continue today. Initially the experiments used a neutron beam and Ramsey separated field technique to measure the Larmor precession frequency [2]. The main limitation of these experiments came from the motional magnetic field if $\mathbf{B}$ and $\mathbf{E}$ were not perfectly parallel along the entire length of the beam apparatus. Later experiments used ultra-cold neutrons that are reflected from many materials and could be stored in bottles for up to $100 \mathrm{sec}$. The most sensitive experiments of this type are performed in Grenoble [7, 8] and Gatchina [9]. In bottle experiments one can greatly reduce the motional magnetic fields since the average neutron velocity is close to zero. The experiments ultimately are limited by counting statistics and magnetic fields produced by the leakage currents. In 1990 the Grenoble group obtained a result $d_{n}=-(3 \pm 5) \times 10^{-26} \mathrm{ecm}$ [7] where the error was dominated by magnetic field systematics. In the later version of the experiment they incorporated a ${ }^{199} \mathrm{Hg}$ co-magnetometer that measured the average magnetic field inside the neutron bottle. That eliminated the magnetic systematic effects, but the statistical error was not reduced. In 1999 the Grenoble group obtained a result $d_{n}=(1.9 \pm 5.4) \times 10^{-26} e \mathrm{~cm}$ after several years of data accumulation. They combined this measurement with their previous result to set a $90 \%$ confidence limit $\left|d_{n}\right|<6.3 \times 10^{-26} \mathrm{ecm}$. However this procedure was criticized in [10] because of large and not completely understood systematic effects in the first measurement. In the mean time the latest results from the Gatchina group were reported in 1996, $d_{n}=(2.6 \pm 4.0 \pm 1.6) \times 10^{-26} e \mathrm{~cm}[9]$ with separate statistical and systematic uncertainties. Their experiment uses two adjacent neutron bottles with opposite electric fields, allowing cancellation of common magnetic field drifts. Considering the combination of all these measurements it is probably justified to set the world limit on the neutron EDM at about $\left|d_{n}\right|<6 \times 10^{-26} e \mathrm{~cm}(90 \%$ C.L.). The Grenoble group is presently working to increase the number of stored neutrons in their apparatus using newer generations of ultra-cold neutron sources.

It was initially thought that EDM searches with a charged particle would be much harder since the particle would accelerate if placed in an electric field. As was pointed 
out by Schiff [11], a neutral atom placed in an electric field would be polarized such that the average electric field felt by each of its charged constituents was equal to zero, at least if one only considered electrostatic interactions. However, soon after discovery of $\mathrm{CP}$ violation in $\mathrm{K}$ mesons it was pointed out by Sandars [12] that in heavy atoms magnetic spin-orbit interactions become quite significant. Careful calculations revealed that in heavy paramagnetic atoms the atomic EDM increases as $d_{a} \propto d_{e} \alpha^{2} Z^{3}$ and can actually be several hundred times larger than the EDM of a bare electron. A succession of experiments using Cs and metastable Xe atoms reduced the electron EDM limit by several orders of magnitude before the current round of $\mathrm{Tl}$ experiments at Berkeley.

The Berkeley experiment $[13,14]$ uses an intense $\mathrm{Tl}$ thermal atomic beam and the standard technique of Ramsey separated fields to measure the Larmor frequency. To combat the motional magnetic fields the experiment uses two oppositely directed atomic beams going through the same set of entrance and exit slits. This allows one to cancel the first order motional field associated with misalignment of $\mathbf{B}$ and $\mathbf{E}$ fields. However, since the motional field has to be suppressed by about 8 orders of magnitude, minute imperfections in the apparatus become significant. The residual effects are caused by slight differences in the trajectories of the counter-propagating atomic beams coupled with magnetic field gradients. Another significant systematic effect comes from Berry's phase [15] that is acquired in the precession of the spins if changes of the quantization axis enclose a finite solid angle. To investigate these systematic effects the most recent version of the experiment [14] uses Na co-magnetometer beams overlapping the $\mathrm{Tl}$ beams. Na has much smaller $Z$ than $\mathrm{Tl}$ and does not have a significant electric dipole moment while having similar sensitivity to systematic effects. It proved challenging to construct an oven capable of simultaneously producing large fluxes of both $\mathrm{Tl}$ and $\mathrm{Na}$ atoms. While $\mathrm{Na}$ atoms were used in systematic studies, their magnetic sensitivity was not sufficient to allow direct subtraction from the Tl data. The experiment also uses pairs of parallel atomic beams propagating in opposite electric field, thereby cancelling common magnetic field drifts and doubling the EDM shift. In total, there are 8 atomic beams with fluxes over $10^{18}$ atoms $/ \mathrm{sec}$. A total of 256 different correlations can be examined to understand various systematic effects. New effects associated with non-adiabatic transitions in and out of the electric field region were discovered in the course of the experiment. The group recently reported their final results, setting a limit on the electron EDM of $\left|d_{e}\right|<1.6 \times 10^{-27} e \mathrm{~cm}$ (90\% C.L.) [14]. This experimental technique has reached its practical limits and the experiment has been disassembled.

Using diamagnetic atoms one can search for an electric dipole moment associated with the nucleus. However, the Schiff theorem again applies implies that the average electric field at the nucleus is equal to zero even if the atom is placed in an electric field. The remaining effect that can be detected is the so-called "Schiff moment", the difference between the distribution of charge and distribution of the electric dipole moment in the nucleus. The Schiff moment induces an atomic EDM that is typically about 3 orders of magnitude smaller than the EDM of the bare nucleus. However, as was pointed out by Khriplovich and co-workers [16], in addition to the EDM of the valence nucleon the Schiff moment has a large contribution from $\mathrm{CP}$-odd nuclear forces that add coherently in the nucleus. As a result, measurements with diamagnetic atoms are quite competitive with other types of EDM experiments.

Most sensitive EDM measurements of this type were performed with ${ }^{199} \mathrm{Hg}$ atoms in Seattle $[17,18] .{ }^{199} \mathrm{Hg}$ is an ideal atom in many regards because it is heavy, has 


\begin{tabular}{|l|l|l|l|}
\hline Experiment & Relation to elementary particles & Uncertainty & Ref. \\
\hline Electron & $d_{T l}=-585 d_{e}$ & $10 \%$ & {$[19]$} \\
Neutron & $d_{n}=0.7 d_{d}-0.2 d_{u}+0.6 e \tilde{d}_{d}+0.3 e \tilde{d}_{u}$ & $50 \%$ & {$[20]$} \\
Mercury & $d_{H g}=-2.8 \times 10^{-4} \mathrm{fm}^{-2} S_{H g}$ & $20 \%$ & {$[22]$} \\
& $S_{H g}=-1.2 e \mathrm{fm}^{3} \bar{g}_{\pi N N}$ & $50 \%$ & {$[23]$} \\
& $\bar{g}_{\pi N N}=20 \mathrm{fm}^{-1}\left(\tilde{d}_{d}-\tilde{d}_{u}\right)$ & Factor of 2 & {$[24]$} \\
& $\Rightarrow d_{H g}=0.007 e\left(\tilde{d}_{d}-\tilde{d}_{u}\right)$ & & \\
\hline
\end{tabular}

Table 1: Relationship between experimental measurements and EDMs of elementary particles. $d_{u}$ and $d_{d}$ are EDMs of the up and down quarks while $\tilde{d}_{u}$ and $\tilde{d}_{d}$ denote their chromo-EDMs.

$I=1 / 2$, resulting in a long coherence time and immunity from quadrupole Stark shifts, and can be probed optically. The atoms are contained in a quartz cell coated with a paraffin coating resulting in a spin coherence time of 100-200 sec. An UV laser at $254 \mathrm{~nm}$ is used for optical pumping of the atoms and detection of their precession frequency. Two cells with oppositely directed electric fields allow cancellation of the magnetic field noise. Based on about 6 months of integration the EDM of ${ }^{199} \mathrm{Hg}$ was measured to be $d\left({ }^{199} \mathrm{Hg}\right)=(-1.06 \pm 0.49 \pm 0.40) \times 10^{-28} e \mathrm{~cm}$, which was interpreted as an upper (95\% C.L.) limit of $\left|d\left({ }^{199} \mathrm{Hg}\right)\right|=2.1 \times 10^{-28} e \mathrm{~cm}$. Recently the experiment underwent a major upgrade, switching from 2 to a stack of $4{ }^{199} \mathrm{Hg}$ cells. The inner two cells have opposite electric fields while the outer cells do not have an electric field and serve to cancel magnetic field gradient noise and detect magnetic fields produced by the leakage currents. The spin lifetime of the cells and other aspects of the experiment have also been improved, increasing the overall sensitivity by a factor of 4 . The experiment is starting to take data.

\section{Interpretation of EDM experiments.}

Since the Standard Model does not predict appreciable EDMs the interpretation of the EDM experiments is relatively straight-forward. Any non-zero result at the present levels of sensitivity would imply new sources of $\mathrm{CP}$ violation. However, to set limits on specific models of CP violation one has to relate the experimental limits to EDMs of fundamental particles. Since all current EDM experiments involve composite particles this requires some calculations based on generally well-known atomic and nuclear physics. The calculations are particularly straight forward for the Tl EDM and involve only atomic theory similar to atomic theory needed for interpretation of parity violation experiments. Detailed many-body perturbation calculation relates the atomic EDM of $\mathrm{Tl}$ to that of the electron with an accuracy of $5-10 \%$ [19].

It is more difficult calculate the constraints imposed by the neutron and ${ }^{199} \mathrm{Hg}$ EDM experiments because they involve hadronic interactions. In addition to electric dipole moments of the quarks these experiments are also sensitive to the so-called chromo-electric dipole moments, which are due to a CP-violating interaction involving a gluon instead of a photon. Using QCD sum rules Pospelov and co-workers recently determined the linear combinations of quark EDMs and chromo-EDMs constrained by these experiments [20, 21]. Table 1 summarizes the relationship between 


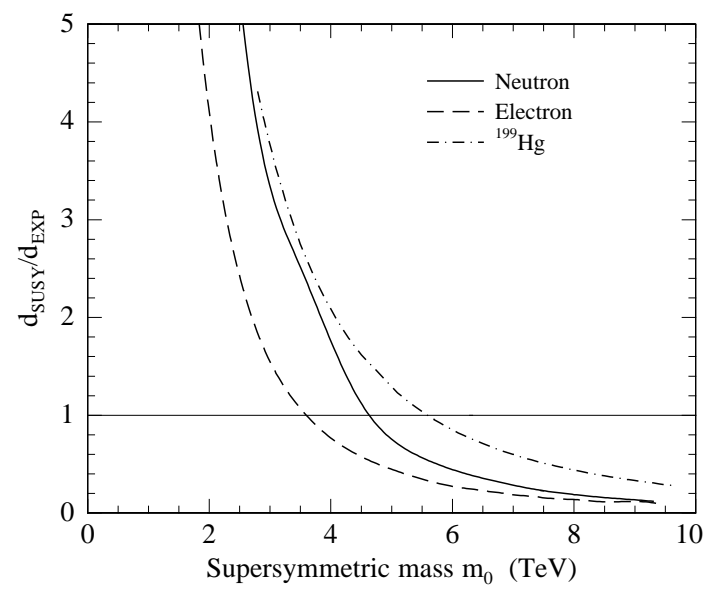

Figure 2: Ratio of supersymmetric EDM prediction to the experimental limit as a function of a universal SUSY mass scale $m_{0}$ assuming $\phi_{S U S Y}=\pi / 2$. Adopted from Ref. [25] including latest limits and calculations.

the experimental limits and EDMs of elementary particles. In the case of ${ }^{199} \mathrm{Hg}$ it involves several steps. ${ }^{199} \mathrm{Hg}$ atomic EDM is related to its Schiff moment based on atomic calculations, most recently refined by Flambaum and co-workers [22]. The Schiff moment is related to the CP-odd pion exchange constant $\bar{g}_{\pi N N}$ using a nuclear shell model [23]. This coupling constant can be used to constrain a CP-violating term in the QCD Lagrangian to be less than $|\bar{\theta}|<2 \times 10^{-10}$, a factor of 3 smaller than the limit from the neutron EDM. The smallness of this term, which is not constrained by any known symmetry, is a long-standing problem in particle physics known as the strong CP problem. Finally, the CP-odd pion exchange constant $\bar{g}_{\pi N N}$ can be related to the chromo-EDMs of the quarks. This calculation has recently been refined by Pospelov [24] resulting in a relaxation of the limits imposed by the ${ }^{199} \mathrm{Hg}$ EDM on the quark chromo-EDMs by a factor of 3 .

Present EDM experiments put very stringent constraints on CP-violating effects beyond the Standard Model. For example in Supersymmetry the natural sizes of EDMs are about a 1000 times larger than the experimental limits. A fermion EDM generally has several contributions of the following form

$$
d_{f} \propto \frac{m_{f}}{M_{S U S Y}^{2}} \sin \left(\phi_{S U S Y}\right)
$$

from exchange of supersymmetric particles. The EDMs can be suppressed below experimental limits by making SUSY CP-violating phases smaller than $10^{-2}-10^{-3}$, making masses of supersymmetric particles larger than several $\mathrm{TeV}$, or arranging cancellation among different EDM contributions to better than 1\%. However, all these scenarios face some theoretical difficulties.

It is difficult to explain the naturalness of small $\mathrm{CP}$-violating phases given that $\mathrm{CP}$ reversal is not an exact symmetry of the Universe. In particular, if CP-violation of the Standard Model is to be unified with other interactions in string-motivated models it 


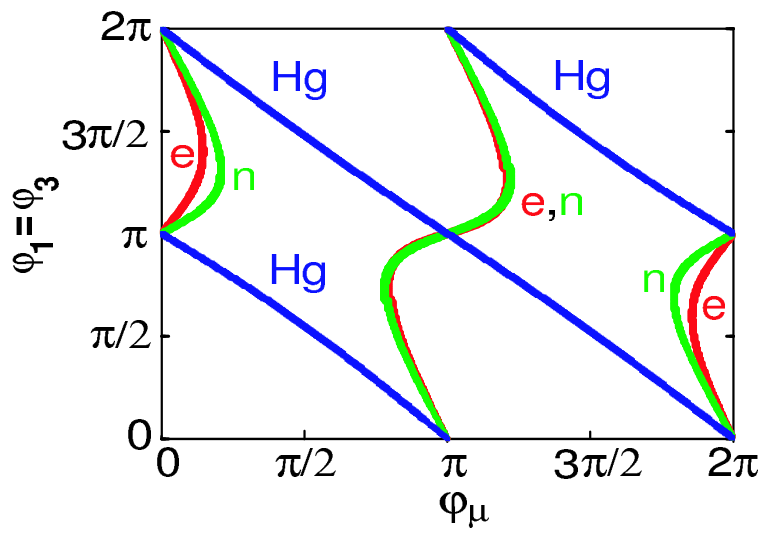

Figure 3: EDM limits constrain SYSY CP-violating phases to be within narrow bands (with a width of about $10^{-2} \mathrm{rad}$ ) indicated on the figure. In some models the bands from neutron and electron limits can be made to overlap [31, 32], allowing both EDMs to be small for certain combinations of phases. The ${ }^{199} \mathrm{Hg}$ experiment constraints a different combination of phases. Thus, the only way to satisfy all experimental constraints is for all SUSY phases to be equal to multiples of $\pi$. Adopted from Ref. $[25]$.

will naturally result in large CP violation in Supersymmetry [26]. Allowing the masses of supersymmetric particles to be very large defeats one of the main motivations for introducing Supersymmetry to stabilize the mass of the Higgs against divergent radiative corrections. Fig. 2 shows the supersymmetric contributions to EDMs compared with experimental limits as a function of a universal SUSY mass scale $m_{0}$. As can be seen, all three experiments place comparable constraints on the supersymmetric mass scale in the range of $4-6 \mathrm{TeV}$ compared with natural mass scale of $100-200 \mathrm{GeV}$. One possible solution is to introduce an inverted mass hierarchy for supersymmetric particles, where the masses of the first two generations of the sfermions are very heavy, while the third generation is light [27]. However, even in this case certain classes of loop diagrams generate a large EDM [28]. Possible cancellations between different EDM contributions to the neutron and electron EDM also have been extensively considered [29, 30, 31, 32, 33, 34]. However, addition of the ${ }^{199} \mathrm{Hg}$ constraint virtually eliminates this possibility, as illustrated in Fig. 3.

While EDM experiments put stringent constraints on CP-violation in Supersymmetry, it is not impossible to circumvent them. Most general SUSY models have over 40 complex phases, so in principle it is possible to arrange for all EDMs to be equal to zero.

\section{New techniques for EDM searches}

A number of new experimental techniques are presently being developed with possibility of improving the EDM limits by several orders of magnitude. One of the promising methods for improving the sensitivity to electron EDM is to use a heavy molecule instead of an atom. As was first pointed out by Sandars [35], polar molecules have 
internal electric fields of about $10^{10} \mathrm{~V} / \mathrm{cm}$ compared to maximum laboratory fields of $10^{5} \mathrm{~V} / \mathrm{cm}$ and can be used to enhance the EDM signal. First experiments of this type used TIF, a diamagnetic molecule, and set limits on the EDM of the proton [36]. Using a paramagnetic molecule with a heavy atom one can be sensitive to the electron EDM and benefit both from heavy atom enhancement and large internal electric fields. Unfortunately, paramagnetic molecules are generally thermodynamically unstable and difficult to produce. Only molecules in the lowest rotational state can be used in the measurement which further reduces the counting rate. The Sussex group succeeded in generating an atomic beam of $\mathrm{YbF}$ and recently reported the first EDM results [37]. After installation of a supersonic atomic beam source they expect to be competitive with the latest Berkeley measurement.

Another approach for molecular EDM experiments is to use an excited paramagnetic state of a diamagnetic molecule. This approach is investigated by DeMille using a metastable $a(1)$ state in $\mathrm{PbO}$ that can be populated with relatively high efficiency by a pulsed laser [38]. The metastable state also has a very small splitting of opposite parity $\Omega$-doublet states and can be fully electrically polarized with a field of only $10 \mathrm{~V} / \mathrm{cm}$. These two factors allow operation of the experiment in a high-density vapor cell resulting in a much higher counting rate. Statistical sensitivity to $d_{e}$ in the range $\left(10^{-29}-10^{-31}\right) e \mathrm{~cm}$ can be expected, depending on the excitation and detection methods. Many classes of systematic effects are also eliminated due to the properties of this molecular system. In addition, the sign of the EDM signal can be reversed by addressing two nearby opposite parity states, which will provide a powerful diagnostic against possible systematic errors.

Recent developments in laser cooling and trapping of atoms also open new avenues for EDM searches. Heavy paramagnetic atoms, such as Cs, can be easily cooled and trapped in far-detuned dipole traps, providing near ideal environment for an EDM experiment and improving the spin coherence times. The number of atoms that has been trapped is not particularly large compared with cell and beam experiments, but they can be polarized and detected with high efficiency. Systematic effects and noise due to the trapping beams have been investigated and appear manageable [39, 40]. It is also possible to use $\mathrm{Rb}$ as a co-magnetometer in the same trap. Several groups are developing laser trapping EDM experiments with a sensitivity goal of about $10^{-29} \mathrm{ecm}$.

A completely different experimental technique for electron EDM search has recently been proposed by Lamoreaux [41]. It is based on detection of a small magnetization in a paramagnetic material induced by the EDM in a strong electric field. Using ultrasensitive SQUID or atomic magnetometers and very low temperatures it may even be possible to achieve sensitivities down to $10^{-33} e \mathrm{~cm}$. A complimentary technique involving detection of a small voltage induced by the EDM in a ferromagnetic material is also being explored [42].

For diamagnetic atoms present experiments are limited by statistics. One way to significantly improve the sensitivity is to increase the number of atoms by switching from a vapor to a liquid. This can be achieved by utilizing liquid ${ }^{129} \mathrm{Xe}$ which has nearly 8 orders of magnitude higher density than the $\mathrm{Hg}$ vapor. It also has a transverse spin coherence time of over $1300 \mathrm{sec}$ [43], the longest ever measured in a liquid, and a very large electrical breakdown strength. Large density of polarized ${ }^{129} \mathrm{Xe}$ spins also results in new types of effects due to their magnetic self-interactions. We find that under certain conditions the magnetic interactions lead to a positive feedback that can be used to amplify the EDM signal. SQUID detectors are used to detect the 
precession of ${ }^{129} \mathrm{Xe}$ spins and as a near-magnetometer. Statistical sensitivity of about $10^{-33} \mathrm{ecm}$ is expected.

Another approach to diamagnetic EDM measurements is to use very heavy radioactive atoms. It was recently pointed out by Flambaum and co-workers [44] that certain isotopes, such as ${ }^{225} \mathrm{Ra},{ }^{223} \mathrm{Ra}$, and ${ }^{223} \mathrm{Rn}$ have an additional enhancement of the Schiff moment by as much as $10^{3}$ due to static octupole deformations and close-lying opposite parity nuclear states. Ra isotopes can be laser cooled and trapped in a dipole trap, while $\mathrm{Rn}$ is a noble gas that can be contained in a cell. Several groups are exploring the feasibility of such experiments.

For improvements in the neutron EDM large increase in the number of ultra-cold neutrons is necessary. This can be achieved by using a superthermal production process, such down-scattering from $11 \mathrm{~K}$ phonons in superfluid He. This allows much more efficient use of cold neutrons produced by a typical moderator. The UCN neutrons can be produced directly in the EDM vessel filled with ${ }^{4} \mathrm{He}$, eliminating extraction losses. Adding a small amount of ${ }^{3} \mathrm{He}$ can serve as a polarizer and detector for precession of the neutrons, as well as a co-magnetometer [45]. Sensitivity levels of $10^{-28} e \mathrm{~cm}$ can be achieved.

\section{Conclusions}

After a 50 year history searches for an electric dipole moment remain an active field and as relevant to understanding of particle physics as ever. Existing experiments put real constraints on CP violation beyond the Standard Model and generate a high level of activity among particle theorists. There are many new ideas on how to improve the sensitivity to EDMs and it will likely remain a very active field.

\section{References}

[1] E. M. Purcell and N. F. Ramsey, Phys. Rev. 78, 807 (1950).

[2] N. F. Ramsey, Rep. Prog. Phys. 45, 95 (1982).

[3] W. Bernreuther and M. Suzuki, Rev. Mod. Phys. 63, 313 (1991).

[4] S. M. Barr, Int. J. Mod. Phys. A 8, 209 (1993).

[5] B. C. Regan, E. D. Commins, C. J. Schmidt, and D. DeMille, Phys. Rev. Lett. 88, 071805 (2002).

[6] R. S. Van Dyck, Jr., P. B. Schwinberg, and H. G. Dehmelt, Phys. Rev. Lett. 59, 26 (1987).

[7] K.F. Smith et al., Phys. Lett. B 234, 191 (1990).

[8] P.G. Harris et al., Phys. Rev. Lett. 82, 904 (1999).

[9] I. S. Altarev et al., Phys. At. Nucl. 59, 1152 (1996).

[10] S. K. Lamoreaux and R. Golub, Phys. Rev. D 61, 051301 (2000).

[11] L. I. Schiff, Phys. Rev. 132, 2194 (1963).

[12] P. G. H. Sandars, Phys. Lett. 14, 194 (1965).

[13] E. D. Commins, S. B. Ross, D. DeMille, and B. C. Regan, Phys. Rev. A 50, 2960 (1994). 
[14] B. C. Regan, E. D. Commins, C. J. Schmidt, and D. DeMille, Phys. Rev. Lett. 88, 071805 (2002).

[15] E. D. Commins, Am. J. Phys. 59, 1077 (1991).

[16] O.P. Sushkov, V.V. Flambaum, and I.B. Khriplovich, Sov. Phys. JETP 60, 873 (1984).

[17] J. P. Jacobs, W. M. Klipstein, S. K. Lamoreaux, B. R. Heckel, and E. N. Fortson, Phys. Rev. A 52, 3521 (1995).

[18] M. V. Romalis, W. C. Griffith, J. P. Jacobs, and E. N. Fortson, Phys. Rev. Lett. 86, 2505 (2001).

[19] Z. W. Liu and H. P. Kelly, Phys. Rev. A 45, R4210 (1992).

[20] M. Pospelov, A. Ritz, Phys. Rev. D 63, 073015 (2001).

[21] T. Falk, K. A. Olive, M. Pospelov, and R. Roiban, Nucl. Phys. B 560, 3 (1999).

[22] V. A. Dzuba, V. V. Flambaum, J. S. M. Ginges, M. G. Kozlov, Phys. Rev. A 66, 012111 (2002).

[23] V. V. Flambaum, I. B. Khriplovich, O. P. Sushkov, Nucl. Phys. A 449, 750 (1986).

[24] M. Pospelov, Phys. Lett. B 530, 123 (2002).

[25] S. Abel, S. Khalil, O. Lebedev, Nucl. Phys. B 606, 151 (2001).

[26] S. Abel, S. Khalil, O. Lebedev, Phys. Rev. Lett. 89, 121601, (2002).

[27] A. G. Cohen, D. B. Kaplan, A. E. Nelson, Phys. Lett. B 388, 588 (1996).

[28] O. Lebedev and M. Pospelov, Phys. Rev. Lett. 89, 101801 (2002).

[29] T. Ibrahim, P. Nath, Phys. Rev. D 58, 111301 (1998).

[30] T. Ibrahim and P. Nath, Phys. Rev. D 61, 093004 (2000).

[31] M. Brhlik, L. Everett, G. L. Kane, J. Lykken, Phys. Rev. Lett. 83, 2124 (1999).

[32] M. Brhlik, L. Everett, G. L. Kane and J. Lykken, Phys. Rev. D 62, 035005 (2000).

[33] M. Brhlik, G. J. Good, G.L. Kane, Phys. Rev. D 59, 115004 (1999).

[34] S. Abel, S. Khalil, O. Lebedev, Phys. Rev. Lett. 865850 (2001).

[35] P. G. H. Sandars, Phys. Rev. Lett. 19, 1396 (1967).

[36] D. Cho, K. Sangster, and E. A. Hinds, Phys. Rev. Lett. 63, 2559 (1989).

[37] J. J. Hudson, B. E. Sauer, M. R. Tarbutt, and E. A. Hinds, Phys. Rev. Lett. 89, 023003 (2002).

[38] D. DeMille et al, Phys. Rev. A 61, 052507 (2000).

[39] C. Chin, V. Leiber, V. Vuletic, A. J. Kerman, and S. Chu, Phys. Rev. A 63, 033401 (2001).

[40] M. V. Romalis and E. N. Fortson, Phys. Rev. A 59, 4547 (1999).

[41] S. K. Lamoreaux, nucl-ex/0109014.

[42] L. R. Hunter, (private communication).

[43] M. V. Romalis and M. P. Ledbetter, Phys. Rev. Lett. 87, 067601 (2001).

[44] N. Auerbach, V. V. Flambaum, and V. Spevak, Phys. Rev. Lett. 76, 4316 (1996).

[45] R. Golub and, S. K. Lamoreaux, Phys. Rep. 237, 1 (1994). 